\title{
The AIRE G228W mutation results in a longer-lasting AIRE-SIRT1 interaction
}

\author{
Jadson C. Santos ${ }^{1 \#}$, Mariangela Dametto ${ }^{2 \#}$, Ana Paula Masson ${ }^{3}$, Vitor M. Faça ${ }^{3}$, \\ Rodrigo Bonacin ${ }^{2}$, Eduardo A. Donadi ${ }^{4}$, Geraldo Aleixo Passos ${ }^{1,5,6, *}$
}

1 Molecular Immunogenetics Group, Department of Genetics, Ribeirão Preto Medical School, University of São Paulo (USP), Ribeirão Preto, SP, Brazil.

2 Renato Archer Information Technology Center (CTI Brazil), Ministry of Science, Technology and Innovation (MCTI), Campinas, SP, Brazil.

3 Department of Biochemistry and Immunology, Ribeirão Preto Medical School, University of São Paulo (USP), Ribeirão Preto, SP, Brazil.

4 Division of Clinical Immunology, Department of Medicine, Ribeirão Preto Medical School, USP, Ribeirão Preto, SP, Brazil.

5 Laboratory of Genetics and Molecular Biology, Department of Basic and Oral Biology, School of Dentistry of Ribeirão Preto, USP, Ribeirão Preto, SP, Brazil.

6 Center for Cell-Based Therapy in Dentistry, School of Dentistry of Ribeirão Preto, USP, Ribeirão Preto, SP, Brazil.

* Corresponding author at: Molecular Immunogenetics Group, Department of Genetics, Ribeirão Preto Medical School, University of São Paulo (USP), Ribeirão Preto, SP, Brazil. E-mail: passos@usp.br

\# Equal contribution. 


\begin{abstract}
The in silico and in vitro binding of a peptide covering a part of the autoimmune regulator (AIRE) SAND domain with the SIRT1 protein provides a powerful model system for studying the mechanism of the dominant SAND G228W mutation, which is the causative of APS-1 autoimmune syndrome. It is known that the mutant G228W AIRE protein accumulates more within the nucleus of cells than its wild-type counterpart does. This accumulation is not physiological and is associated with loss of AIRE function. However, the precise molecular mechanism that leads to AIRE accumulation is not yet known. AIRE works as a tetramer and interacts with partner proteins to form the "AIRE complex" that pushes RNA Pol II stalling in the chromatin of medullary thymic epithelial cells. Under normal conditions, the SIRT1 protein temporarily interacts with AIRE and deacetylates Lys residues of the SAND domain. Once AIRE is deacetylated, the binding with SIRT1 is undone, allowing the complex to proceed downstream. Here, we integrate molecular modeling, docking, dynamics, and surface plasmon resonance approaches to compare the structure and energetics of binding/release between AIRE G228 (wild-type) or W228 (mutant) peptides to SIRT1. We find that the proximity of G228W mutation to a $\mathrm{K}$ aminoacid residue in the SAND domain promotes a longer-lasting AIRE-SIRT1 interaction. The lasting interaction might cause a delay in the AIRE SAND domain to be released from the SIRT1 catalytic site, which might cause accumulation of the defective AIRE mutant protein in the nuclei of cells.
\end{abstract}

Key words AIRE, SAND domain, SIRT1 interaction, APS-1 mutation mechanism, molecular dynamics

\title{
Significance
}

This report reveals the mechanism of the pathogenic and dominant G228W mutation in the AIRE SAND domain. The G228W mutation is found in APS-1 syndrome patients, and it is critical to understand the molecular basis of loss of self-representation, a challenging aspect for immunology. Through modeling, molecular dynamics, and protein binding kinetics, we found that the G228W mutation leads to a stronger physical interaction between the AIRE SAND domain and the SIRT1 protein when compared to the equivalent wild-type segment. The short-term consequence of this stronger interaction is that the release of the AIRE-SIRT1 binding is slower. This might explain the reason that cells carrying the G228W mutation accumulate AIRE protein in their nuclei. This finding reveals with precision the AIRE-SIRT1 binding and the molecular mechanism of the human AIRE G228W mutation.

\section{Introduction}

The human autoimmune regulator (AIRE) gene located at chromosome 21q22.3 is $11.9 \mathrm{~kb}$ long, spans 14 exons, and encodes an mRNA with 1635 nucleotides, which in turn encodes a 545 aminoacid protein (Aaltonen et al., 1994; Nagamine et al., 1997; Blechschmidt et al., 1999). Nearly 145 mutations have been found along the AIRE gene, from single-nucleotide mutations to large deletions (Wolff and Oftedal, 2019; Besnard et al., 2021; Human Gene Mutation Database available at https://www.hgmd.cf.ac.uk/ac/). Mutations in the AIRE gene are related to polyendocrinopathy autoimmune-candidiasis-ectodermal dystrophy (APECED) (Finnish-German APECED Consortium 1997; Nagamine et al., 1997), also known as polyglandular autoimmune syndrome type 1 (APS1) (OMIM entry \# 240300). 
Most mutations that cause APS-1 are considered to be inherited in an autosomal recessive manner. Exceptions are a group of mono-allelic mutations, clustered within the zinc finger domain of the first plant homeodomain (PHD1) (Oftedal et al., 2015; Wolff and Oftedal, 2019), and a mutation in the SAND domain, G228W, which follows a dominant inheritance pattern (Cetani et al., 2001; Ilmarinen et al., 2005; Su et al., 2008).

The AIRE protein is a transcriptional regulator in medullary thymic epithelial cells (Passos et al., 2018; Perniola, 2018; Giraud and Peterson, 2019). During its action in the chromatin, it interacts with several protein partners forming the "AIRE complex," being SIRT1 one of the most important because it is an enzyme that deacetylates K residues in the SAND domain, promoting the AIRE function (Incani et al., 2014; Abramson et al., 2010; Abramson and Husebye, 2016).

AIRE has a molecular weight of $56 \mathrm{KDa}$ and is structured in five domains and four LXXLL motifs, the CARD domain, caspase activation, and recruitment domain, which is essential for AIRE dimerization; the NLS domain, nuclear localization signal that mediates the AIRE migration from the cytoplasm to the nucleus, the SAND domain involved in promoting protein-protein interactions and chromatin binding, and two PHD domains (PHD1 and PHD2) that function as histone code readers, interacting and supporting in chromatin decondensation (Anderson et al., 2016; Kumar et al., 2001; Perniola et al., 2014; Giraud and Peterson, 2019).

The G228W mutation in the SAND domain was the first dominant mutation associated with APS-1 syndrome (Cetani et al., 2001; Ilmarinen et al., 2005; Su et al., 2008; Wolff and Oftedal, 2019). Experimental data indicate that the AIRE G228W mutant protein targets the cell nucleus similarly to its wild-type counterpart. Still, cells that contain only one of the mutant alleles cannot promote the proper transcription of genes responsible for promoting immune tolerance (Ilmarinen et al., 2005). As a result, individuals who carry the G228W mutation present the APS-1 syndrome (Wolff and Oftedal, 2019).

In addition to chromatin binding, the SAND domain may also have a regulatory role in the homomultimerization of AIRE (Ramsey et al., 2002a; Halonen et al., 2004). In this domain, chemical modifications occur, such as the acetylation of lysine residues essential for the AIRE function (Saare et al., 2012). Lysines from the SAND domain are also deacetylated by the SIRT1 protein, an NAD+ dependent histone and non-histone protein deacetylase (Vaquero et al., 2004; Bheda et al., 2016).

Protein acetylation influences the protein's stability, location, and affinity to DNA or proteins (Glozak et al., 2005). The acetylation and deacetylation status along AIRE can regulate its stability (Incani et al., 2014). The deacetylation of lysine residues in the SAND domain activates the function of AIRE, influencing downstream peripheral tissue antigen gene regulation, which is essential for central tolerance induction (Chuprin et al., 2015; Saare et al., 2012).

The G228W mutation occurs at a position located at six aminoacid residues from one of the acetylated lysine residues (K222) and is further deacetylated by SIRT1 (Saare et al., 2012). Despite the characterization of the G228W mutation in the SAND domain 
of AIRE and its evident association with the aggressive autoimmune manifestation of the APS-1 syndrome, the reason for its dominant character and the molecular mechanism of its pathogenesis is still elusive.

In this study, we raised the hypothesis that the proximity of the G228W mutation to a $K$ residue, whose subsequent deacetylation activates the function of the AIRE protein, may interfere with the interaction between the SAND domain the SIRT1 protein. To test this, we integrate in silico molecular modeling, molecular dynamics, and in vitro surface plasmon resonance approaches to elucidate the structure and energetics of binding/release between AIRE G228 (wild type) W228 (mutant) peptides to SIRT1 protein.

Overall, results show that the G228W mutation leads to a stronger physical interaction between the peptide segment of the AIRE SAND domain surrounding the mutant residue and the SIRT1 catalytic site compared to the equivalent wild-type segment, which elucidates the intrinsic mechanism of this mutation.

\section{Materials and Methods}

\section{Protein structure homology modelling}

We used the SWISS-MODEL workspace for protein modeling available at (http://swissmodel.expasy.org/workspace). The aminoacid sequence in the FASTA format of the SIRT1 target protein was used as input. The SWISS-MODEL algorithms performed BLASTp and provided the best homology models based on Global Model Quality Estimation (GMQE) and Qualitative Model Energy ANalysis (QMEAN) statistical parameters. The GMQE provided the quality by combining the targettemplate alignment properties and estimating a range between 0 and 1. The scoring function of QMEAN consisted of a linear combination of structural descriptors, which is related to the high-resolution X-ray crystal structure and estimates a $Z$-score scheme.

\section{Molecular docking}

Molecular docking analysis of the previously modeled AIRE SAND domain, wild-type AIRE SAND domain or G228W mutant AIRE SAND domain, and the SIRT1 protein (PDB code 4I5I), was performed to study the stability of protein-protein interaction.

The ClusPro 2.0 protein-protein docking server (Alekseenko et al., 2020) (available at https://cluspro.org/) was used. In the ClusPro server, the rigid body docking phase uses the PIPER docking program (https://www.schrodinger.com/products/piper), which relies on the Fast Fourier Transform (FFT) correlation approach. PIPER represents the interaction energy between two proteins using an expression of the formula $E=w 1$ Erep $+w 2$ Eattr $+w 3$ Eelec $+w 4 E D A R S$

Where, E rep and $E$ attr denote the attractive and repulsive contributions to the van der Waals interaction energy, and $E$ elec represents the electrostatic energy.

$E D A R S$ is a pairwise structure-based potential; it primarily represents desolvation contributions, i.e., free energy change by removing the water molecules from the 
interface. The coefficients $w 1, w 2, w 3$, and $w 4$ define the molecular weights of the corresponding aminoacid residues.

\section{Systems setup and molecular dynamics simulations}

The 3D structures of the complexes formed with AIRE wild-type or with AIRE mutant peptide and SIRT1 generated in the docking section as above mentioned were used as initial configurations for the molecular dynamics simulations. In this work, calculations were performed to verify the stability and affinity of the complexes.

To mimic the interaction between the two peptides and SIRT1 protein, the K222 residue was acetylated using the parameters settled as in the AMBER Molecular Dynamics Package (https://ambermd.org/) for this residue. ACK and the connectivity library file for K222 residue was generated by tleap.

The two systems were solvated using the TIP3P water model and neutralized with four $\mathrm{Na}$ + ions in the tleap tool implemented in AMBERTOOLS21 (https://ambermd.org/AmberTools.php). The whole system was truncated in a box with a $15 \AA$ between the edges of the periodic box and the molecules analyzed. An $8 \AA$ cutoff Particle Mesh Ewald (PME) method was used to treat the long-range electrostatic interactions. The AMBER molecular dynamics software and the ff19SB force field were used to prepare the systems and run the simulations.

Before running, the complexes were submitted to minimization in two stages using the steepest descent method followed by a conjugate gradient. In the first stage, the elements, peptides, and SIRT1 were fixed, and the position of the water molecules was minimized. After that, the whole system was minimized. A restrained thermalization phase with the micro-canonical ensemble (NVE) was performed, incrementing the temperature every $0.1 \mathrm{~K}$ until it reached $300 \mathrm{~K}$ using Langevin thermostat and SHAKE algorithm (available at https://ambermd.org/) to constrain hydrogen bonds.

The production dynamics were carried out at constant pressure (NPT ensemble) with a time step of $2 \mathrm{fs}$. The total simulation time for each complex is $2700 \mathrm{~ns}$, and frames were saved every $20 \mathrm{ps}$. The analyses were done in the last $2650 \mathrm{~ns}$ after the systems had been equilibrated. All the calculations shown in the current work were done using AMBERTOOLS21 scripts. The visualization programs used to create the figures of the molecules were VMD for LINUXAMD64, version 1.9.3, and PYMOL (available at https://pymol.org/2/).

\section{Surface plasmon resonance}

The surface plasmon resonance (SPR) experiments were performed in a Biacore model T200 equipment (GE Life Sciences). For the immobilization of human SIRT1 (Sigma-Aldrich S8846), a CM5 sensor chip S series, which has carboxylic groups available for covalent coupling, was used. All solutions used were purchased from the equipment supplier (GE Life Sciences). The procedures described here were performed 
automatically with the Biacore instrument, using pre-established programs for binding amines present in peptides or proteins.

Briefly, the carboxylic groups present on the CM5 sensor chip surface were activated with EDC + NHS, forming the NHS ester reactive intermediate, which, after washing with HBS-N, readily reacted with amines dissolved in the immobilization solution. The human SIRT1 ligand was dissolved in $10 \mathrm{mM}$ sodium acetate $\mathrm{pH} 4.0$ plus 20 uM NAD+ (beta-nicotinamide adenine dinucleotide hydrate Sigma-Aldrich, N1636), which was immobilized onto the Biacore sensor chip according to the manufacturer's instructions.

To detect interactions between SIRT1 and AIRE WT or AIRE G228W mutant SAND domain peptides, different concentrations of peptides were injected onto the CM5 chip with immobilized SIRT1 protein. Peptide samples were injected sequentially from lowest to highest concentration $(0,10,15,20,50,100,200$, and $300 \mu \mathrm{M})$ and a contact time of $300 \mathrm{~s}$, a $20 \mu \mathrm{l} / \mathrm{min}$ flow and dissociation time of $1200 \mathrm{~s}$. The surface was regenerated between samples by injecting $2.0 \mathrm{M}$ glycine $\mathrm{pH} 2.0(30 \mathrm{~s}, 30 \mu \mathrm{l} / \mathrm{min})$ followed by injection of $2.0 \mathrm{M} \mathrm{NaCl}(30 \mathrm{~s}, 30 \mu \mathrm{l} / \mathrm{min})$. The stabilization period between injections after regeneration was $200 \mathrm{~s}$.

The respective sequences of the twenty-aminoacid residues peptides comprising positions 211 to 230 of the SAND domain of the human AIRE protein are as follows, AIRE WT (IQQVFESGGSKKKCIQVGGEF) and mutant AIRE G228W mutant (IQQVFESGGSKKEIQVGWEF). The $\mathrm{K}$ residue overlined was acetylated or not, depending on the experiment. The AIRE wild-type or AIRE G228W mutation data is available through GenBank NCBI (https://www.ncbi.nlm.nih.gov/) accession numbers (NM_000383.4): c.682G $>\mathrm{T}$ G [GGG] > W [TGG], coding sequence variant autoimmune regulator (NP_000374.1): p.Gly228TrpG (Gly) > W (Trp) missense variant, DbSNP (https://www.ncbi.nlm.nih.gov/snp/rs121434257) and ClinVar (https://www.ncbi.nlm.nih.gov/clinvar/variation/3313/).

Kinetic data were processed and analyzed with the Biacore T200 version 3.0 software (GE Healthcare). Data obtained with the different concentrations of AIRE WT peptide were combined in the same graph. Similarly, data with varying concentrations of the AIRE G228W mutant peptide were combined for comparative visualization.

\section{Results}

\section{Homology molecular modeling of the AIRE SAND domain}

As of the date of completion of this work on August 24, 2021 the threedimensional structure of the entire human AIRE SAND domain were not available in public databases. To obtain it, we use the SwissModel tool for modeling proteins through homology.

We use the SAND domain sequence of the human SP100 protein (PDB code 1h5p) for the modeling, which features $39.62 \%$ sequence identity to the human AIRE SAND domain. The SP100 protein SAND domain conserves the SKKCIQ aminoacid sequence present in the AIRE SAND domain, which corresponds to the accurate interaction site between SAND and the SIRT1 protein. 
Thus, it was possible to obtain a three-dimensional (3D) structure of the wildtype (WT) AIRE SAND domain. Through mutagenesis using PyMol software, we got the 3D structure of the AIRE SAND domain carrying the G228W mutation. We observed that the G228W substitution did not cause misfolding in the mutant SAND domain structure (figure 1).

\section{WT (G228) SAND domain of AIRE Mutant (W228) SAND domain of AIRE}
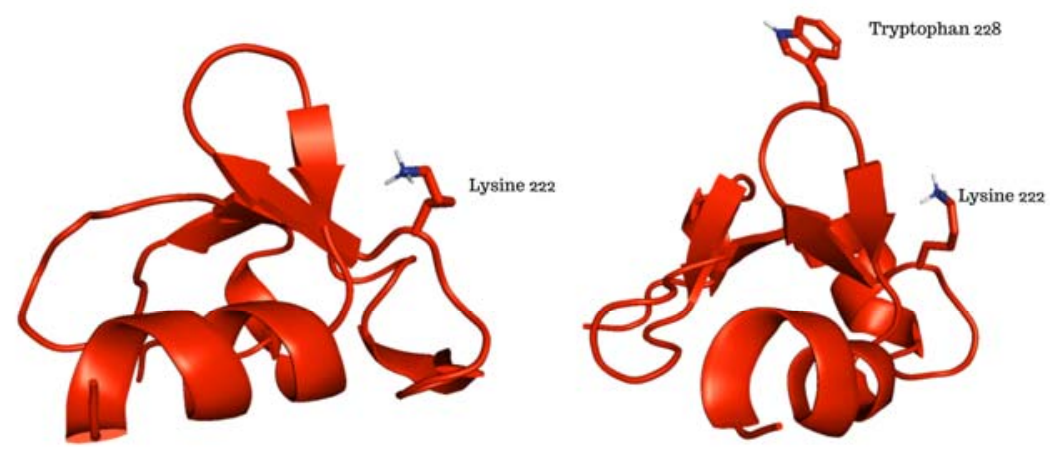

Figure 1. Molecular modeling of the G228 wild-type (left) or mutant W228 AIRE SAND domain (right) as obtained through homology with the human SP100 SAND domain.

\section{Blind molecular docking shows that SAND W228 mutant residue was targeted to the SIRT-1 catalytic residue}

Through blind molecular docking, it was possible to observe that was an affinity between the AIRE SAND domain with the catalytic site (H363 residue) of the SIRT-1 protein, even without any previous targeting or chemical modification of the K 222 residue, which is a target of the SIRT1 protein (Figures 2 - 4).

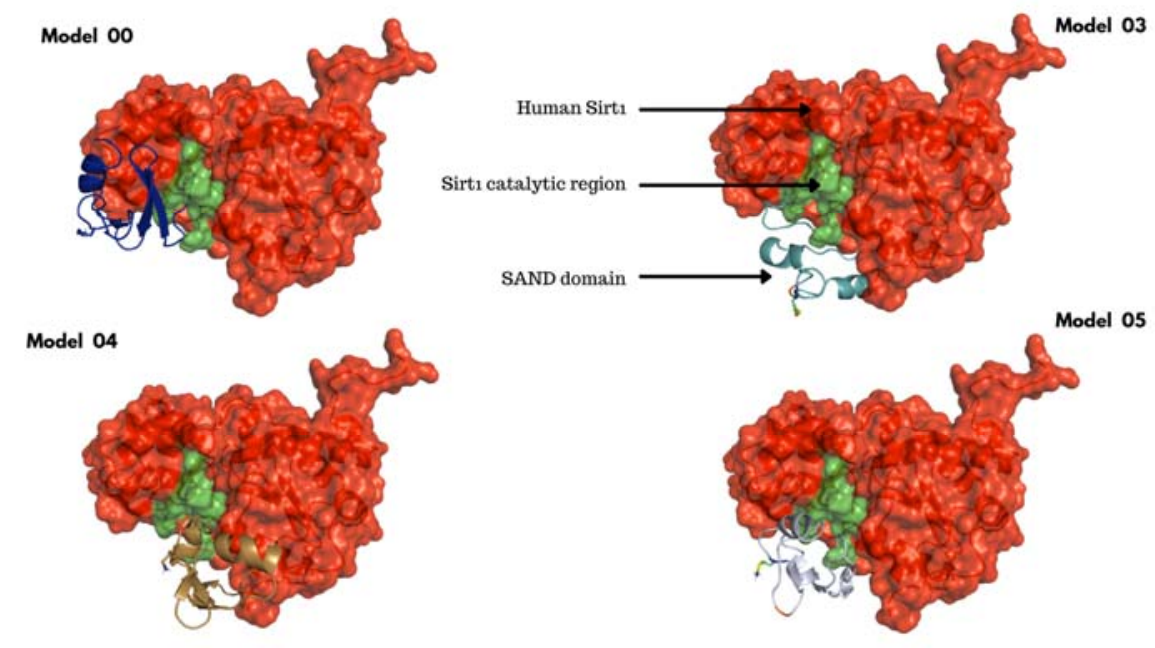


Figure 2. Molecular models were obtained from blind molecular docking between the wild-type AIRE SAND domain with the SIRT1 catalytic domain. The models showed correspond to the best representatives from ten docking cluster outputs. Model 00 corresponds to the closest to the complex's native structure according to the ClusPro 2.0 server.
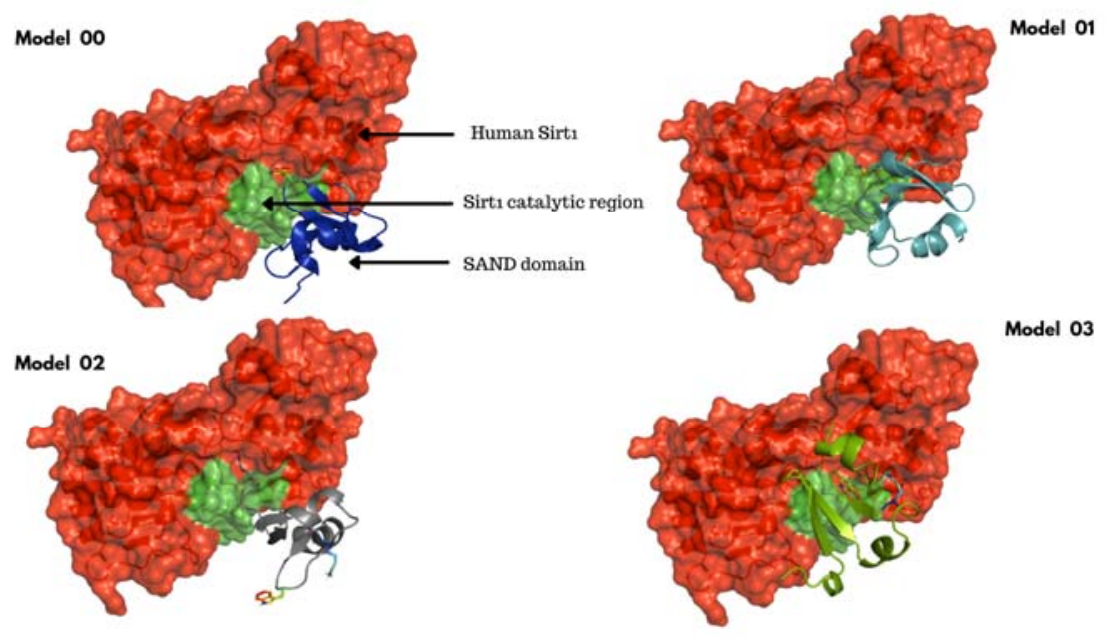

Figure 3. Molecular models were obtained from blind molecular docking between the mutant W228 AIRE SAND domain with the SIRT1 catalytic domain. The models showed correspond to the best representatives from ten docking cluster outputs. Model 00 corresponds to the closest to the complex's native structure according to the ClusPro 2.0 server.

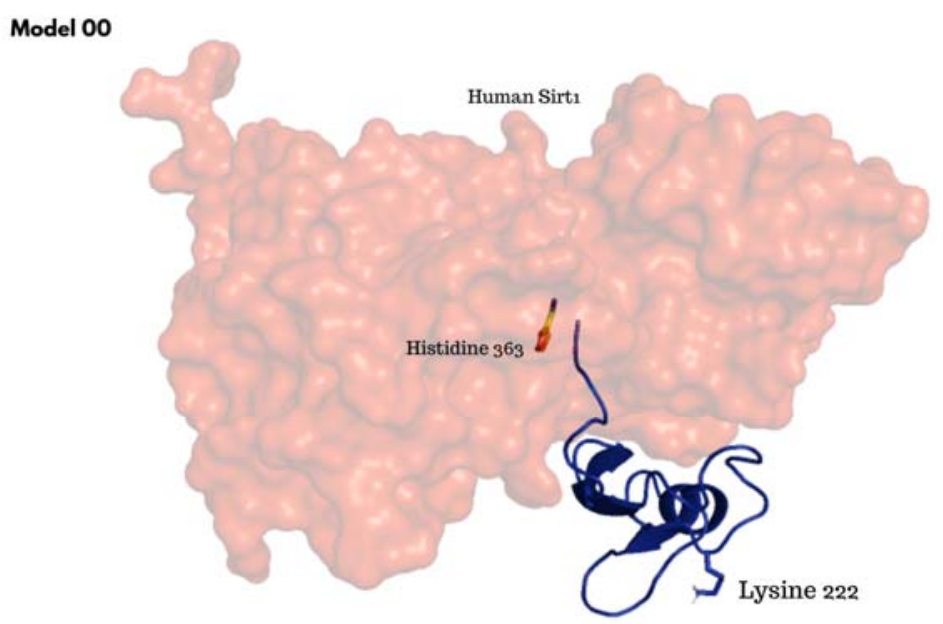

Figure 4. Docking output of wild-type AIRE SAND domain showing the K222 residue from the AIRE side interacting with the H363 catalytic residue from the SIRT1 side. 
Comparing the structural conformations, we observed that the SAND domain mobilizes the W228 mutant residue that interacts with SIRT1 H363 catalytic residue in 7 out of 10 docking analyses (Figure 5).

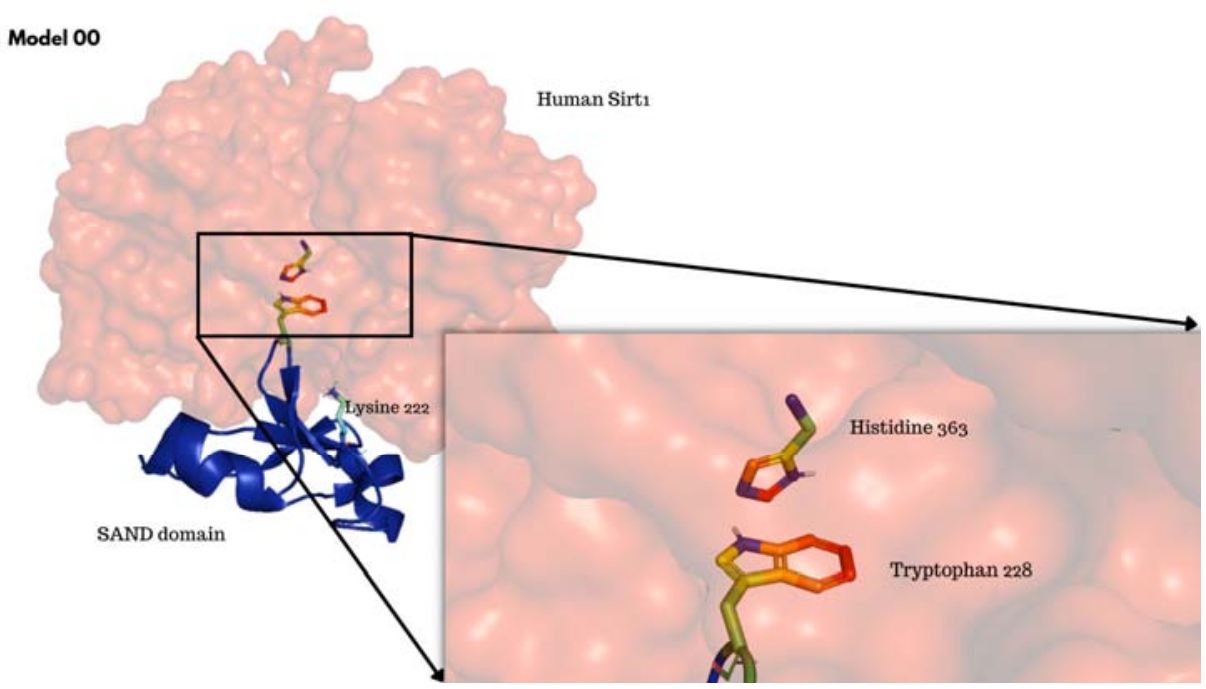

Figure 5. Docking output of mutant W228 AIRE SAND domain showing the W228 residue from the AIRE side interacting with the H363 catalytic residue from the SIRT1 side. Blind docking evidenced the targeting of mutant residue W228 to catalytic residue H363 of SIRT1.

\section{Molecular dynamics}

The molecular dynamics results show that the complex formed between WT SAND domain with SIRT-1 is slightly less stable along the simulation time than the complex formed between SAND G228W mutant. Nevertheless, the complex involving the G228W mutant domain keeps stable without significant jumps in the RMSD, suggesting a more favorable conformation with SIRT1 (Figures 6-8). 


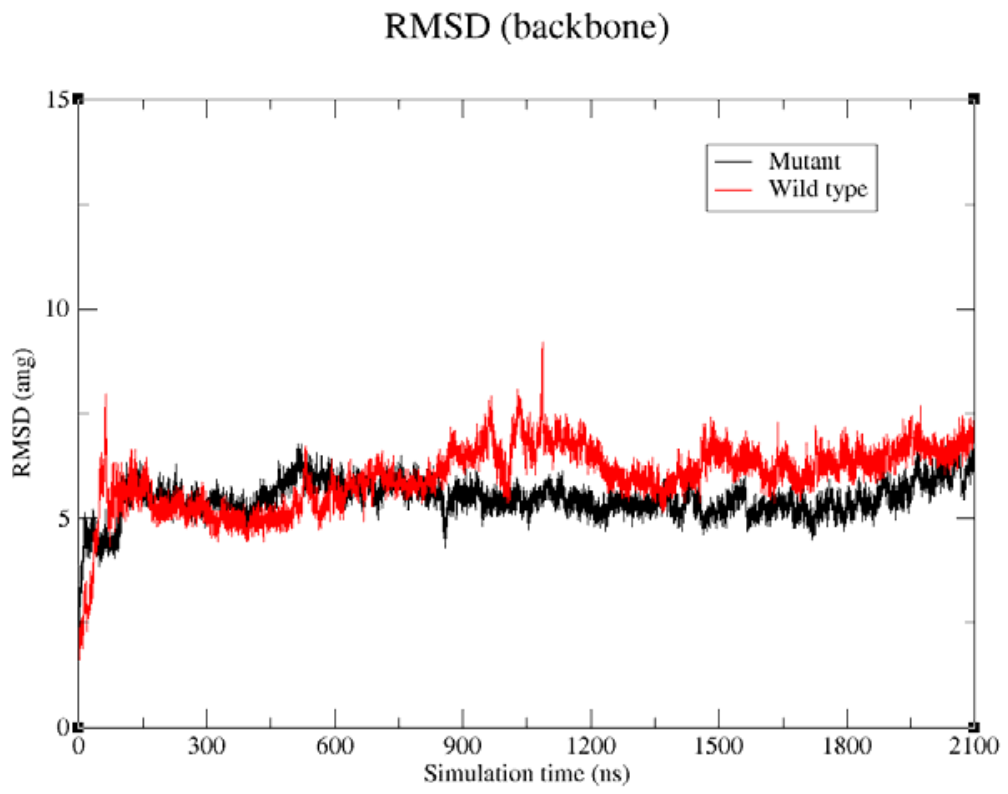

Figure 6. Molecular dynamics of the wild-type (red) or mutant W228 (black) AIRE SAND domain with the SIRT1 protein.

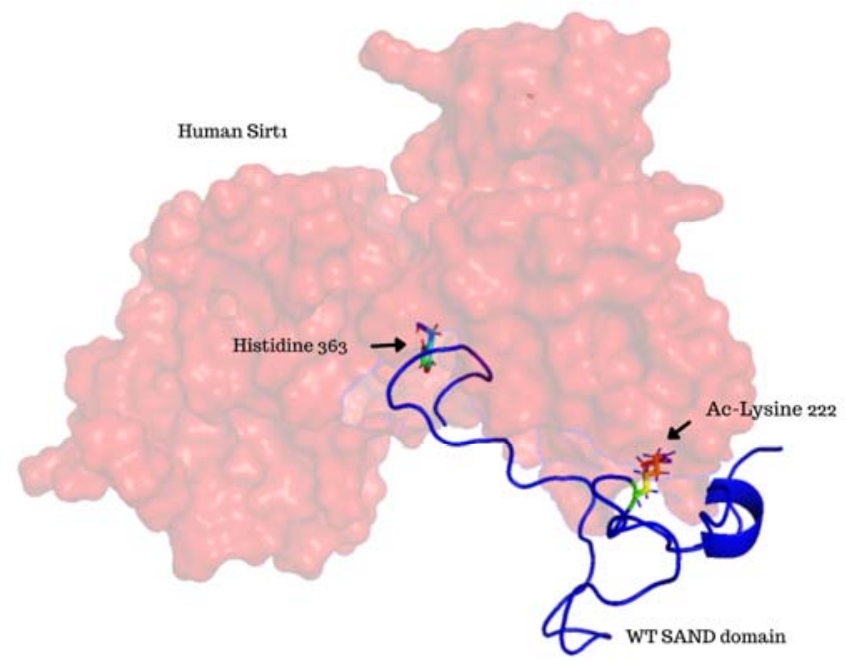

Figure 7. Molecular dynamics structure formed between the wild-type AIRE SAND domain and SIRT1 protein. Note that the acetylated K222 residue from the AIRE side is not directed to the SIRT1 H363 catalytic residue during the analyzed dynamics time (1.0 microsecond). 


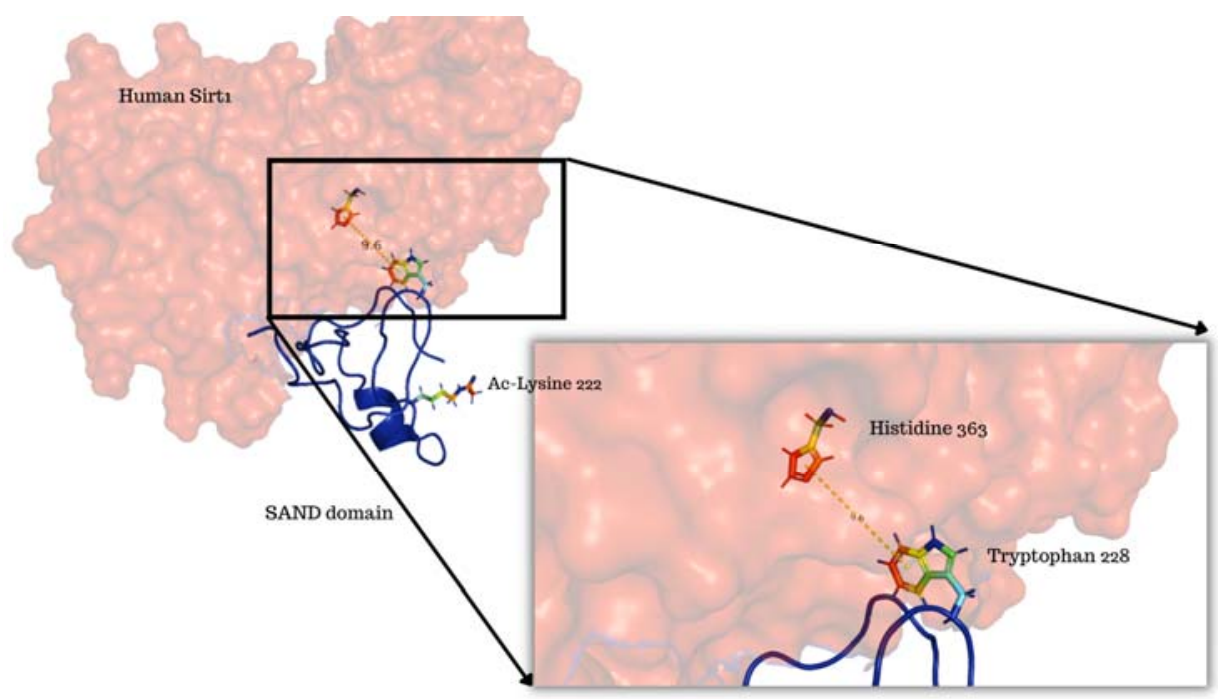

Figure 8. Molecular dynamics structure formed between the mutant W228 AIRE SAND domain and SIRT1 protein. Note that at 1.0 microsecond of dynamics, the mutant residue W228, seen from the side of AIRE, remained close to the catalytic H363 residue of SIRT1.

Furthermore, we evaluated in these complexes the distances in $\AA$ that the SIRT1 catalytic residue (H363) of forms with AIRE. The results show that in the SAND AIRE domain, the mutant aminoacid residue W228 remains more closely interacted with the SIRT1 catalytic residue compared to the WT G228 residue (Figure 9 and Table 1). Altogether, the computational analyses show better interaction between the AIRE SAND domain and SIRT1 when the SAND G228W mutation is present.

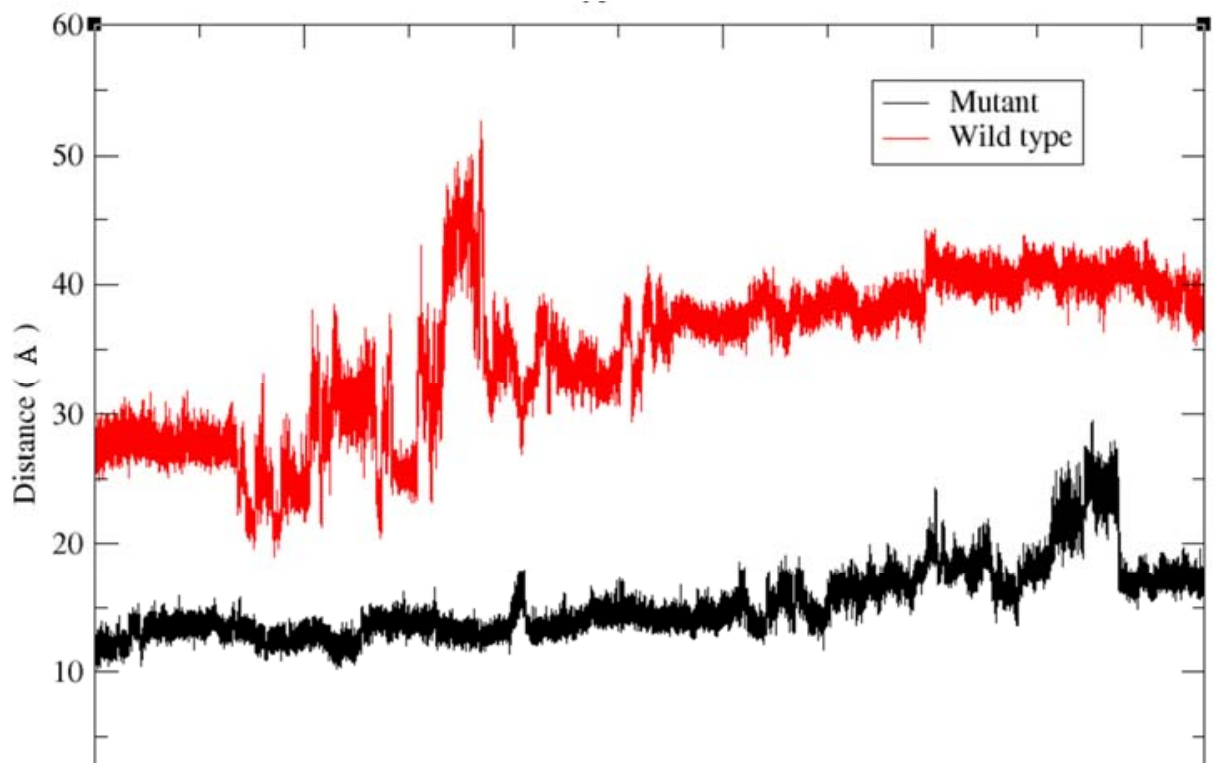


Figure 9. Distances between aminoacid residues G228 (wild-type) or W228 (mutant) of the SAND domain of AIRE with the SIRT1 protein. Note that residue W228 (mutant) is more stable in the interaction than residue G228 (wild-type).

Surface plasmon resonance indicates that SAND G228W mutation modifies the interaction kinetics of AIRE with SIRT-1

Surface plasmon resonance (SPR) results indicated a differential association and dissociation dynamics between the peptides WT SAND G228 or mutant SAND W228 with the SIRT1 protein. The interaction kinetics showed that the peptide WT SAND G228 has greater association capacity. Nevertheless, it showed a greater dissociation capacity (concentration range 10 to $100 \mathrm{uM}$ ). The interaction between SAND WT G228 and SIRT1 was almost wholly undone after 1200 seconds of measurement (Figure 10A).

Conversely, the mutant SAND W228 peptide was comparatively less able to associate with SIRT-1, but once associated, it remained stable throughout the measurement (concentration range 10 to $100 \mathrm{uM}$ ) (Figure 10B). These results experimentally validate the computational modeling and molecular dynamics. 


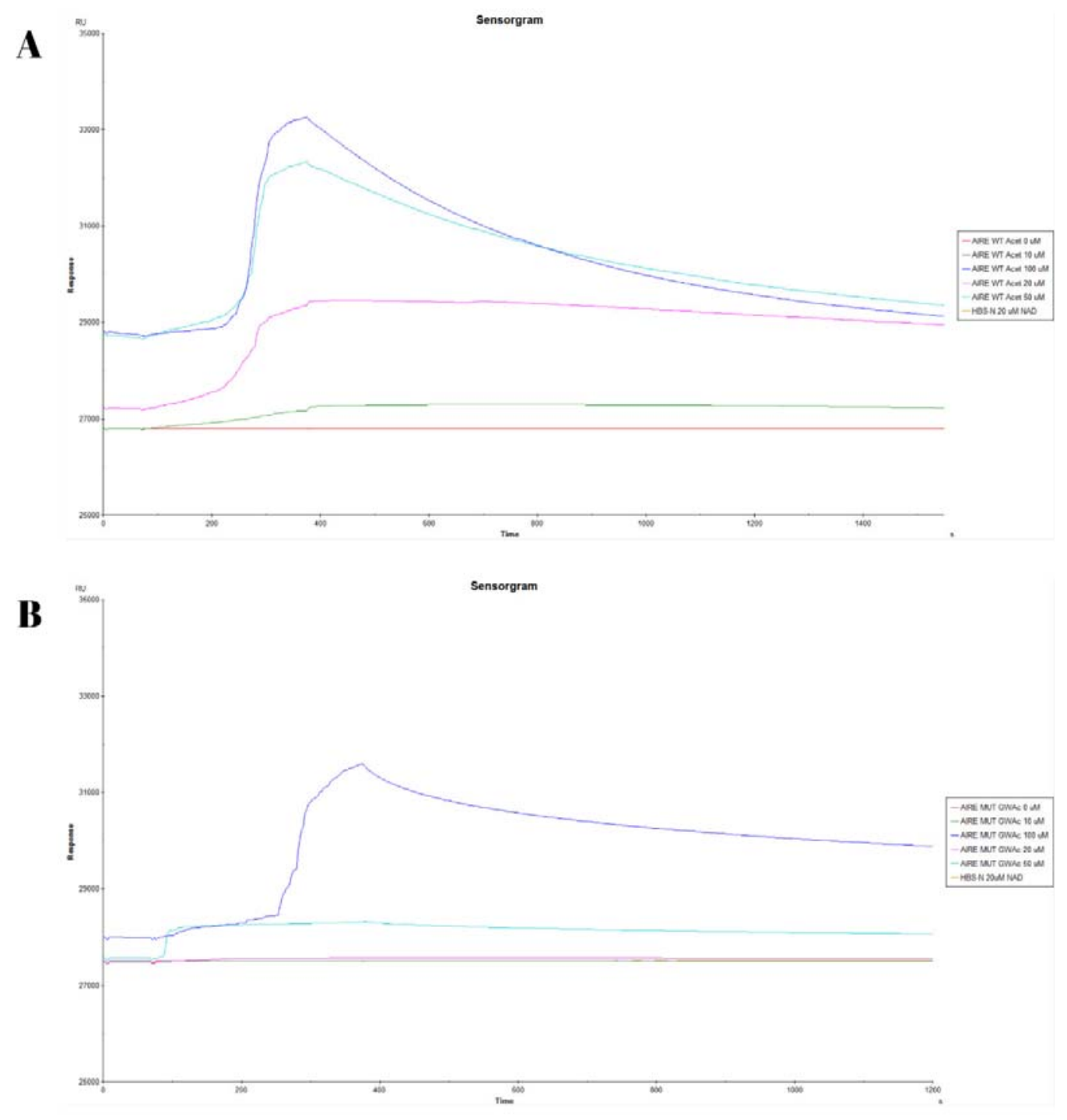

Figure 10. Surface plasmon resonance sensorgrams for binding of the AIRE SAND domain with SIRT1 protein immobilized onto sensor chip. A) Wild-type AIRE SAND domain peptide, B) W228 mutant AIRE SAND domain peptide. The peptides were assayed at various concentrations (10 to $100 \mathrm{uM})$.

Table 1. Contact analysis in the region of interaction between wild-type (G228) or mutant (W228) AIRE SAND domain with SIRT1 protein at 3-angstrom cutoff. 


\begin{tabular}{|r|c|c|c|c|}
\hline & Contact & Wild Type & Average distance & Stdev \\
\hline $\mathbf{1}$ & ARG:274-GLU:207 & 359 & 2.8 & 0.0938 \\
\hline 2 & GLN:345 - ALA:191 & 198 & 2.82 & 0.0965 \\
\hline \multicolumn{7}{|c|}{ Mutant } \\
\hline
\end{tabular}

\section{Discussion}

In this work, we demonstrate that the dominant G228W mutation that occurs in the SAND domain of the human AIRE protein (Cetani et al., 2001) entails a stronger and longer-lasting interaction with the SIRT1 partner, which is a deacetylase (Vaquero et al., 2004; Bheda et al., 2016).

Although there is a more significant interaction between the mutant SAND domain and the catalytic site of SIRT1, the kinetics of the molecular interaction process, that is, the rate of association and dissociation, differs from the kinetics involving the wild type SAND domain. This could be the crucial point that destabilizes the catalytic action of SIRT1 for AIRE protein de-acetylation. This finding is essential to understand the molecular mechanism of G228W mutation better.

The autosomal dominant mutations of the AIRE gene identified so far are grouped in the SAND and PHD1 domains, while the recessive mutations are dispersed throughout the gene/protein (Anderson and Su 2016; Wolff and Oftedal, 2019).

According to Anderson and Su (2016), identifying autosomal dominant mutations in AIRE raises some interesting questions. The first one is related to the frequency of these mutations in the general population. Anderson et al. (2014) found that 1 in 1000 individuals in the general population may have a point mutation in the PHD1 domain. The G228W mutation in the SAND domain of AIRE was the first dominant mutation to be associated with the APS-1 syndrome (Cetani et al., 2001; Ilmarinen et al., 2005; Wolff and Oftedal, 2019).

The further question is how these mutations influence the function of AIRE at the molecular level. Halonen et al. (2004) and Ilmarinen et al. (2005) observed that the G228W mutation quantitatively altered the nuclear location of the AIRE protein and the mutant form accumulates more in the cell nucleus and severely loses its transactivating property. Furthermore, Anderson and $\mathrm{Su}$ (2016) demonstrated that this mutation interferes with the localization of the AIRE protein in the transcription sites of peripheral tissue antigens (PTAs), reducing its expression.

Here we raise a third question, namely, whether the G228W mutation interferes with binding with one of the partner proteins of the AIRE complex.

Although there is not yet a 3D structure for the complete human AIRE protein available, we could access the 3D structure for the AIRE SAND domain by homology folding to the human SP100 protein SAND domain, which is available. 
We noted that the $\mathrm{G} 228 \mathrm{~W}$ replacement domain did not result in a misfolding of AIRE's 3D SAND structure, which could in itself alter the protein functionality. The wild-type or mutant AIRE SAND domain did not show differences in their respective 3D structures. This may be due to the segment's characteristics in which the G228W mutation is present, which is a flexible loop region (SAND amino acid residues 226228). Therefore, the G228W mutation must involve another mechanism.

Then we asked if the mutation studied could interfere with AIRE binding to one of its partner proteins since AIRE needs to be de-acetylated to initiate the downstream cascade of interactions until it pushes RNA Pol II stalling in the chromatin. We point to SIRT1, which is the deacetylase enzyme that integrates the AIRE complex.

To obtain an interaction model between the AIRE SAND domain (WT or G228W mutant) with the SIRT1 and assess the affinity of the mutant SAND domain, we used the ClusPro 2.0 blind molecular docking tool. For this, the structures of the WT SAND domain, the G228W mutant output from the homology modeling, and the structure of the SIRT-1 catalytic domain (PDB code 4I5I) were used. We observed that the SAND domain mobilizes the W228 mutant aminoacid residue that interacts with SIRT1 H 363 catalytic residue.

The AIRE protein plays its role in the cell nucleus as a tetramer (Abramson et al., 2010; Incani et al., 2014), in which the SAND domain of each subunit has $\mathrm{K}$ residues that SIRT1 deacetylates. Ilmarinen et al. (2005) performed a functional analysis of the mutations that occurred in the AIRE SAND domain and concluded that the negative effect of the heterozygous G228W mutation is related to its binding the AIRE WT protein, thus preventing the protein from forming complexes required for transactivation. In this complex, mutant subunit-containing acetylated $\mathrm{K}$ residue could form a hybrid tetramer unable to promote AIRE transactivation activity.

Saare et al. (2012) found that AIRE with mutations mimicking acetylated lysine 243 or 253 residues in the SAND domain reduced transactivation activity and accumulated as nuclear bodies, while mutations mimicking non-acetylated lysines were functionally similar to WT AIRE.

For SIRT1 to exert its enzymatic activity, it depends on the aminoacid residues near the acetylated lysines in the AIRE SAND domain (Garske et al., 2006). Accordingly, we hypothesized that the G228W mutation close to acetylated K residues could interfere with the SIRT1 interaction since it is an enzyme necessary to convert the acetylated to the non-acetylated and functional form of AIRE. Though not assessed here, a consequence of this would be the interference in the deacetylation of K residues.

Studying the R247C mutation also present in the AIRE SAND domain, Abbott et al. (2018) identified that the mutant protein retains the ability to form complexes with wild-type AIRE and localize to the nucleus. However, despite the normal nuclear localization, R247C reduces the activation of downstream transcription through dominant-negative inhibition. The R247C mutation occurs close to other $\mathrm{K}$ residues (K243 and K253) in the SAND domain that SIRT1 could deacetylate. This could be an interference factor and a cause for the negative impact of the R247C mutation.

The molecular dynamics results with the AIRE SAND domain (WT or G228W mutant) showed that docking with acetylated K 222 and after $1 \mu$ s of simulation, the 
WT domain was not able to form a stable structure, in which K 222 would interact with H 363 from the catalytic site of SIRT1.

On the other hand, the G228W mutant domain exhibited stable interaction with the SIRT-1 catalytic site at H 363 residue. To verify the stability of both simulations and the molecular interaction, the atomic position of the backbone atoms relative to the reference structure was calculated using root mean square deviation (RMSD). This confirms the hypothesis raised in this study in which we consider that AIRE G228W mutation interferes with SIRT1 binding, making it stronger and more stable.

Finally, these findings were validated using surface plasmon resonance, whose approach allowed us to evaluate the association/dissociation kinetics of the AIRE SAND domain, WT, or encompassing the G228W mutation peptides which interact with the SIRT-1 protein. We were able to observe that both peptide types can bind to SIRT1. However, the one that harbors the mutation was docked longer.

Together, results provide a precise molecular basis for clarifying how the mutant G228W AIRE protein loses its function.

\section{Supplementary material}

Video 1: AIREW228SIRT1.mov : https://drive.google.com/file/d/1h2TdYfyTpnACZY6jLCJORZEsZoL6-OL/view?usp=sharing

Video 2: AIREG228SIRT1.mov : https://drive.google.com/file/d/123Od2jwRKHz0ZVpalxoyp2YA3_IwJXB/view?usp=sharing

\section{Video legends}

Video 1: Full simulation $(2.1 \mu \mathrm{s})$ of the interaction between SIRT-1 and the wild type AIRE SAND domain. Proteins are shown in Cartoon representation, SIRT-1 is colored in cyan and the wild type AIRE SAND domain is colored in yellow. Key residues described to be important for the interaction between the two molecules are the Histidine (pink) of the SIRT-1 molecule and the acetylated Lysine (green) of the wild type AIRE SAND domain. The atoms of these residues are represented by solid van der Waals spheres. The residue in orange is a Glycine (in the AIRE domain) that is mutated by a Tryptophan in our experiments. This complex showed a weaker interaction when compared to the mutated complex during the entire simulation, which agrees with the result from the surface plasmon resonance experiments (see Movie 2).

Video 2: Full simulation $(2.1 \mu \mathrm{s})$ of the interaction between SIRT-1 and the mutated AIRE SAND domain. The graphic representations of SIRT-1 and the wild type AIRE SAND domain is Cartoon. The key residues Histidine and the acetylated Lysine are in the solid van der Waals spheres representation. The Tryptophan residue, which was mutated by the Glycine, is colored in white. This mutated Tryptophan residue is interacting with the Histidine (SIRT-1) during the entire simulation, which suggests a reason for the longer dissociation time observed from the surface plasmon resonance experiments when compared to the wild type AIRE SAND domain.

\section{Acknowledgments}


This work was funded by the following Brazilian Research Support Agencies, Fundação de Amparo à Pesquisa do Estado de São Paulo (FAPESP, grants \# 17/10780-4 to GAP and EAD, and \# 2019/23448-3 to ACM-C), Conselho Nacional de Desenvolvimento Científico e Tecnológico (CNPq, grants \# 305787/2017-9 to GAP, and 302060/2019-7 to EAD) and Coordenação de Aperfeiçoamento de Pessoal de Nível Superior (CAPES, Financial Code 001).

\section{References}

Abbott J. K., Huoh Y. S., Reynolds P. R., Yu L., Rewers M., Reddy M., et al. Dominant-negative loss of function arises from a second more frequent variant within the SAND domain of autoimmune regulator (AIRE). J Autoimmun. 2018; 88:114-20.

Abramson J., Giraud M., Benoist C., Mathis D. Aire's partners in the molecular control of immunological tolerance. Cell. 2010; 8;140(1):123-35.

Abramson J., Husebye E. S. Autoimmune regulator and self-tolerance - molecular and clinical aspects. Immunol. Rev. 2016; 271:127-140.

Anderson M. S., Su M. A. AIRE expands: new roles in immune tolerance and beuond. Nat. Rev. Immunol. 2016; 16: 247-258.

Alekseenko A., Ignatov M., Kozakov D. Protein-protein and protein-peptide docking with ClusPro server. Methods Mol. Biol. 2020; 2165:157-174.

Anderson M. S., Su M. A. AIRE expands: new roles in immune tolerance and beyond. Nat Rev Immunol 2016; 16:247-58.

Besnard M., Padonou F., Provin N., Giraud M., Guillonneau C. AIRE deficiency, from preclinical models to human APECED disease. Dis. Model. Mech. 2021; 14)2): dmm046359.

Bheda P., Jing H., Wolberger C., and Lin H. The Substrate Specificity of Sirtuins. Annual Review of Biochemistry. 2016; 85, 405-429.

Blechschmidt K., Schweiger M., Wertz K., Poulson R., Christensen H. M., Rosenthal A., et al The mouse Aire gene: comparative genomic sequencing, gene organization, and expression. Genome Res 1999; 9:158-66.

Case D. A. et al. AMBER. 2019, University of California, San Francisco.

Cetani F., Barbesino G., Borsari S., Pardi E., Cianferotti E., Pinchera A., Marcocci C. A novel mutation of the autoimmune regulator gene in an Italian kindred with autoimmune polyendocrinopathy-candidiasis-ectodermal dystrophy, acting in a dominant fashion and strongly cosegregating with hypothyroid autoimmune thyroiditis J. Clin. Endocrinol. Metab. 2006; 86:4747-4752. 
Chuprin A., Avin A., Goldfarb Y. et al. The deacetylase Sirt1 is an essential regulator of Aire-mediated induction of central immunological tolerance. Nat Immunol. 2015; $16: 737-745$.

Dai H., Case A., Riera T. et al. Crystallographic structure of a small molecule SIRT1 activator-enzyme complex. Nat Commun. 2015; 6:7645.

Finnish-German APECED Consortium (1997) An autoimmune disease, APECED, caused by mutations in a novel gene featuring two PDH-type zinc-finger domains. Nat. Genet. 1997; 17(4): 399-403.

Garske A. L., Denu J. M. SIRT1 top 40 hits: use of one-bead, one-compound acetylpeptide libraries and quantum dots to probe deacetylase specificity. Biochemistry. 2006; 10;45(1):94-101.

Glozak M. A., Sengupta N., Zhang X., Seto E. Acetylation and deacetylation of nonhistone proteins. Gene. 2005; 363:15-23.

Giraud M., Peterson P. The autoimmune regulator (AIRE) gene, the master activator $\mathrm{f}$ self-antigen expression in the thymus. In: Thymus Transcriptome and Cell Biology. (Passos GA, Editor). 2019; Springer-Nature Switzerland AG.

Halonen M., Kangas H., Rüppell T., Ilmarinen T., Ollila J., Kolmer M., Vihinen M., Palvimo J., Saarela J., Ulmanen I., Eskelin P. APECED-causing mutations in AIRE reveal the functional domains of the protein. Hum Mutat. 2004; 23:245-257.

Humphrey, W., Dalke, A. and Schulten, K.VMD - Visual Molecular Dynamics. J. Molec. Graphics. 1996; 14.1:33-38

Ilmarinen T., Eskelin P., Halonen M., Ruppell T., Kilpikari R., Torres G. D. et al. Functional analysis of SAND mutations in AIRE supports dominant inheritance of the G228W mutation. Hum Mutat. 2005;26(4):322-331. doi: 10.1002/humu.20224.

Incani F., Serra M., Meloni A., Cossu C., Saba L., Cabras T., Messana I., Rosatelli M. C. AIRE acetylation and deacetylation: effect on protein stability and transactivation activity. J Biomed Sci. 2014; 27:21-85.

Kozakov D. The ClusPro web server for protein-protein docking. Nat Protoc. 2017; 12(2):255.

Kumar P. G., Laloraya M., Wang C. Y., Ruan Q. G., Davoodi $\square$ Semiromi A., Kao K. J. et al. The autoimmune regulator (AIRE) is a DNA $\square$ binding protein. J Biol Chem 2001; 276:41357-64.

Nagamine, K. et al. Positional cloning of the APECED gene. Nat. Genet., 1997; 17 (4), pp. 393-398.

Oftedal B. E., Hellesen A., Erichsen M.M., Bratland E., Vardi A., Perheentupa J. et al. Dominant mutations in the autoimmune regulator AIRE are associated with common organ-specific autoimmune diseases. Immunity. 2015; 42:1185-1196. 
Passos G. A., Speck-Hernandez C. A., Assis A. F., Mendes-da-Cruz D. A. Update on Aire and thymic negative selection. Immunology 2018; 10-20.

Perniola R., Musco G. The biophysical and biochemical properties of the autoimmune regulator (AIRE) protein. Biochim Biophys Acta 2014; 1842:326-37.

Ramsey C., Bukrinsky A., Peltonen L. Systematic mutagenesis of the functional domains of AIRE reveals their role in intracellular targeting. Hum Mol Genet. 2002; 11:3299-3308.

Saare M., Rebane A., Rajashekar B., Vilo J., Peterson P. Autoimmune regulator is acetylated by transcription coactivator CBP/p300. Exp Cell Res. 2012; 15:1767-78.

Samanta U., Pal D., Chakrabarti P. Packing of aromatic rings against tryptophan residues in proteins. Acta Crystallogr D Biol Crystallogr. 1999; 55:1421-1427.

Su M. A., Giang K., Zumer K., Jiang H., Oven I. et al. Mechanisms of an autoimmunity syndrome in mice caused by a dominant mutation in Aire. J. Clin. Invest. 2008; 118(5): 1712-1726.

Vaquero A., Scher M., Lee D., Erdjument-Bromage H., Tempst P., Reinberg D. Human SirT1 interacts with histone $\mathrm{H} 1$ and promotes formation of facultative heterochromatin. Mol. Cell. 2004; 16: 93105.

Wolff S. B., Oftedal B. E. Aire mutations and autoimmune diseases. In: Thymus Transcriptome and Cell Biology. (Passos GA, Editor). 2019; Springer-Nature Switzerland AG. 